Article

\title{
Fourier Coefficients Applied to Improve Backscattered Signals in A Short-Range LIDAR System
}

\author{
Iván Gómez-Arista ${ }^{1}$ * , José A. Dávila-Pintle ${ }^{2}$, Nancy Montalvo-Montalvo ${ }^{2}$, \\ Abel A. Rubin-Alvarado ${ }^{2}$, Yolanda E. Bravo-García ${ }^{2}$ and Edmundo Reynoso-Lara ${ }^{2}$ \\ 1 CONACYT-INAOE, Luis Enrique Erro \# 1, Tonantzintla, Puebla 72840, Mexico \\ 2 Meritorious Autonomous University of Puebla, Puebla 72570, Mexico; \\ jose.davila@correo.buap.mx (J.A.D.-P.); montalvo.ncym@gmail.com (N.M.-M.); \\ arubinwb@hotmail.com (A.A.R.-A.); yolanda.bravog@correo.buap.mx (Y.E.B.-G.); \\ edmundo.reynoso@correo.buap.mx (E.R.-L.) \\ * Correspondence: ivan.goar@gmail.com
}

Received: 11 November 2019; Accepted: 4 February 2020; Published: 27 February 2020

\begin{abstract}
Light Detection and Ranging (LIDAR) is a remote sensing technique that measures properties of backscattered light in order to obtain information of a distant target. This work presents a method to improve the signal-to-noise ratio by $8 \mathrm{~dB}$ with respect to the direct detection of the backscattered signal of a LIDAR system. This method consists of the measurement of the Fourier coefficients of the LIDAR signal, which is possible thanks to the novel coupling of a sequential equivalent time base sampling (SETS) circuit and a conventional lock-in amplifier that allows to measure the Fourier coefficients of the LIDAR signal, the results are comparable to noise elimination using Empirical Mode Decomposition. The feasibility of the proposal is demonstrated experimentally with mist. The method can be used to different applications of elastic-scattering LIDAR under the conditions of the devices for applied the phase sensitive detection.
\end{abstract}

Keywords: LIDAR; signal-to-noise ratio; phase sensitive detection

\section{Introduction}

LIDAR systems are applied to navigation systems of mobile robots [1], to terrestrial and airborne laser scanning of landslides [2], sensing of the atmosphere [3] and the monitoring emission rates and gas concentration levels [4]. According to the application, the LIDAR systems can be divided into two groups: elastic-scattering LIDAR and inelastic-scattering LIDAR, in the first, laser radiation is scattered by atoms without undergoing a change in its wavelength. In the second one, the frequency of the incident photon is different from the frequency of the scattered photon, in both cases, the pulse of the signal contains all the target information [3-6]. There are many analysis methods of the LIDAR signal, for example: in the inelastic-scattering LIDAR system there are Differential Optical Absorption Spectrometer (DOAS) and Fourier Transform Infra-red Spectrometer (FTIR) [7]. In the case of elastic-scattering LIDAR there are numerical analysis with Rayleigh o Mie scattering theory in accordance with the size of the particle. In this case, measurements of intensity of the backscattered signal in which the amplitude of the signal is usually analyzed, selected points of the backscattered signal are different in accordance with designs of LIDAR systems, and additionally, criteria used in the measurements of each system do not necessarily coincide [8]. For the above mentioned, LIDAR applications have opened impressive advances on their data acquisition systems and develop new methods in signals processing. For the processing of the backscattered signal of the target, the detected signal is very small because it decays in a quadratic manner according to distance, additionally, background noise such as solar radiation and atmospheric turbulence is added to the signal. Also, 
detectors commonly used, as avalanche photodiodes and photomultipliers, the noise of its dark current and thermal noise are added to the total noise present in LIDAR measurements. In order to improve the measurements of the backscattered signal of the LIDAR and its subsequent analysis, at the signal can be applied some technique of denoising as a temporal or spatial average, smoothing, Fourier Transform, Wavelet Transform or Empirical Mode Decomposition (EMD). Low pass filters are applied in the technique of average, this method has a wide bandwidth and poor cut-off frequency [9]. The smoothing of the signal is a simple and convenient method although it decreases the temporal resolution of the pulse. To apply the Fourier and Wavelet transforms, users must have prior knowledge of its adjusting parameters which is based on the experience and this is the main limitation of these methods [10]. The EMD is a nonlinear technique that decomposes a given signal into a finite series of components, called intrinsic mode functions, however, the reconstruction of the signal lacks explicit criteria to determine which modes are relevant. Further, partial reconstruction often omits some useful information that is present in the discarded modes [9-11].

In this work is presented another approach to processes the LIDAR backscattered signal, taking advantage of the periodicity of the LIDAR signal, because, it is well known that for periodic signals any change in their amplitude can be measured through the change in any of their Fourier coefficients. Fourier coefficients (FC) can be measured with great precision using a lock-in; this task is trivial for low frequency signals, however for LIDAR signals it is much more complicated due to the very short duration of LIDAR pulses, which have a duration below of nanoseconds and a conventional lock-in is not fast enough. In order to accomplish this measurement, we present a novel way to measure the Fourier coefficients with the use of sequential equivalent time base sampling (SETS) circuits and a conventional lock-in amplifier which allows to have an improvement of the signal-to-noise ratio of the backscattered signal. In order to show that FC measurement offers to be a method of analyzing the LIDAR signal an experiment with a short-range LIDAR system was carried out using a chamber filled with mist as scattering media, the behavior of the backscattered signal of an elastic-scattering LIDAR, by measuring the first two coefficients of its Fourier series [12], is presented.

\section{Experiment}

In order to simulate a scattering cloud, Figure 1 shows a schematic view of the experiment using a LIDAR system and a chamber of mist. The LIDAR used in this work is a Laser Rangefinder System (LRS) OSLRF-01 that includes a pulsed laser, detector, optics and sequential-equivalent-time-sampling circuits (SETS). The pulsed laser wavelength is $850 \mathrm{~nm}$, pulse width $<30 \mathrm{~ns}$, pulse frequency $<16 \mathrm{kHz}$ and average energy per pulse $<0.15 \mathrm{~nJ} / 200 \mathrm{~nJ}$. The OSLRF-01 works on the time-of-flight principle, ideal to measure path length through a given medium and with a range of distance of $18 \mathrm{~m}$. Also, it scales signals that travel at speed of light at the speed of sound by a factor of approximately 881,000 times. The chamber for mist is a cylinder whose transverse section is $7.5 \mathrm{~cm}$ diameter in the plane $\mathrm{X}-\mathrm{Y}$ and its length (L) can be adjusted from $150-300 \mathrm{~cm}$ in the direction $\mathrm{Z}$, there was an inlet near to one edge of the chamber where the mist was pumped by a humidifier, near to the opposite edge there was collocated a ventilation outlet, in order to have a flow of mist. To avoid laser light reflection towards photodetector of the LIDAR by the structure of the chamber, transparent caps were placed on ends of the chamber at an angle of 20 degrees with respect to the axis of propagation of laser beam and an opaque screen was placed at the exit of the chamber in order to avoid reflections of others objects. The mist into the chamber is filled and controlled through an Ultrasonic Humidifier (UH). With the aim to apply the technique of sensitive phase detection with Fourier coefficients (FC), the backscattered signal from mist is measured from the detector of the LRS. In the time domain, the signal of the detector is recorded to the input signal of a dual Lock-in SR530 using an oscilloscope Tektronix, the SETS was set for a period T $=26 \mathrm{~ms}$, and the Fourier coefficients are obtained with the same lock-in with $\omega=2 \pi / T$. It is important to mention that both measurements are obtained at the same time in order to analyze the signal-to-noise ratio of the LIDAR signal. 


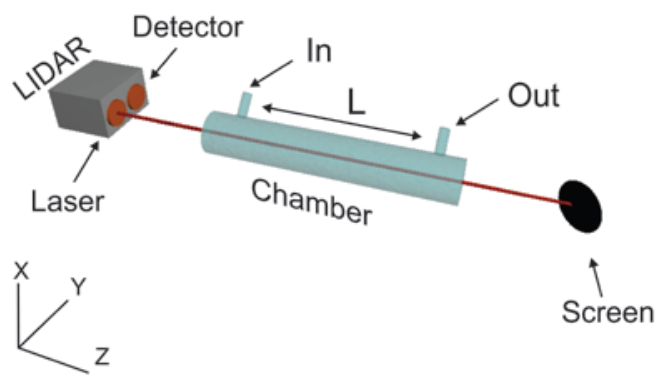

Figure 1. Schematic view of the experiment.

\section{Results and Discussion}

Figure 2 shows the temporal evolution of first coefficient $C_{1}$ and second coefficient $C_{2}$ of Fourier from the lock-in while the backscattered pulse cross through the mist; these coefficients correspond to fundamental frequency $(\omega)$ and second frequency $(2 \omega)$ when the length of the chamber is of $150 \mathrm{~cm}$. The curves show the switching from on to off from the UH in order to show the transition of the mist into the chamber. Description of the process can be divided in three parts. In the first part (I) starting from $t=0 \mathrm{~s}$ to $t=64 \mathrm{~s}$, curves show the amplitude of the FC since the chamber is empty until it is filled with mist when the UH is turned on. The second part (II), from $64 \mathrm{~s} \leq \mathrm{t} \leq 129 \mathrm{~s}$ the chamber is filled with mist and there is constant flow through all chamber; in the third part (III) corresponding to time $t \geq 129 \mathrm{~s}$ the curves show the free flow of the mist when the UH is turned off. The coefficients $C_{1}$ and $\mathrm{C}_{2}$ have a time difference at the beginning of the filling of the chamber, this is due to the fact that the lock-in takes each of the coefficients in a commutated way, but in part II, the curves show that the time of commutated is irrelevant because the chamber is filled with mist and they show the turbulence of the mist inside of the chamber. Part II of Figure 2 is analyzed in order to validate the improvement of the LIDAR signal.

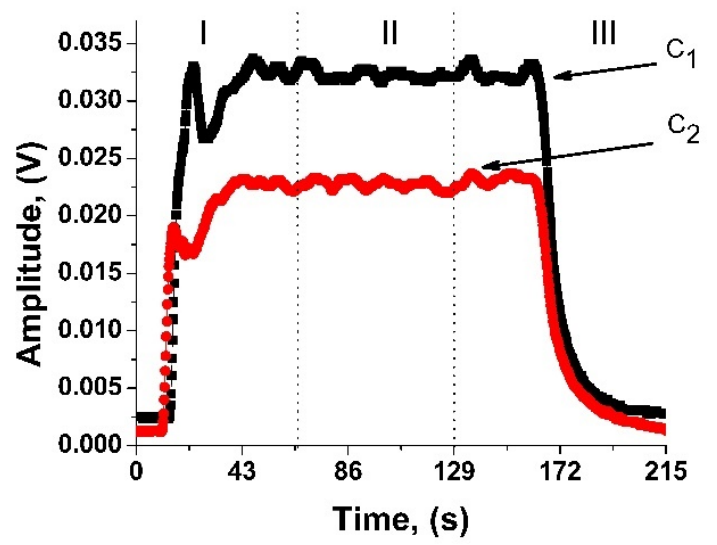

Figure 2. $C_{1}$ and $C_{2}$ Fourier coefficients obtained with the lock-in from mist for a chamber length of $150 \mathrm{~cm}$.

Figure 3 shows the pulse $\left(f(t)\right.$, blue line) that correspond to the input signal of the lock-in when $C_{1}$ is measured in the part II from Figure 2. Theoretical Fourier coefficients $C_{T 1}$ and $C_{T 2}$ are calculated by adjusting $f(t)$ with a Gaussian function $\left(f^{*}(t)\right.$, shown as a dashed red line in Figure 3 for $\left.C_{1}\right)$. 


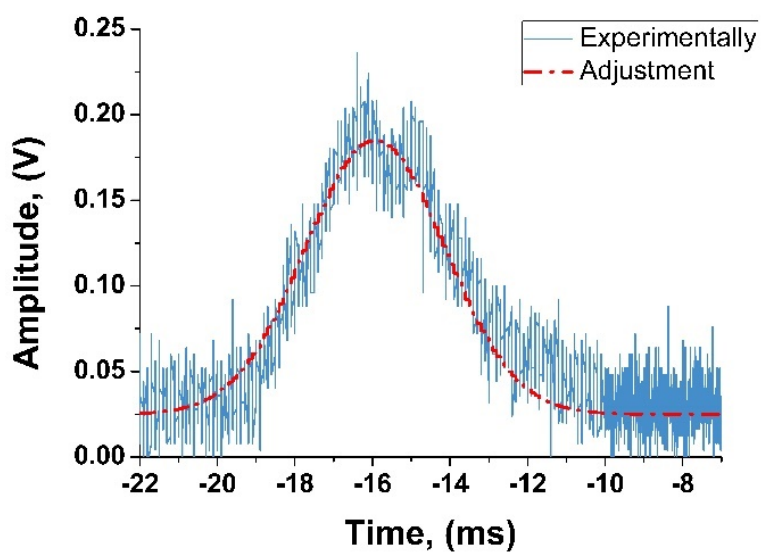

Figure 3. Adjustment of the pulse in the input signal of the lock-in using a Gaussian function.

For a Gaussian periodic signal, its Fourier coefficients is $[13,14]$

$$
C_{T n}=\exp \left(-\frac{\sigma^{2}\left(n \omega_{0}\right)^{2}}{2}\right)
$$

where $\sigma^{2}$ is the variance, $n$ is the $n$ th-coefficient and $C_{T n}$ decreases exponentially with the square of $\mathrm{n}$. In this work, the lock-in just can measure the first two Fourier coefficients directly and, in accordance to $C_{T n}$, the first two coefficients contribute with more power to the LIDAR signal. The results are $\mathrm{C}_{\mathrm{T} 1}=0.032 \mathrm{~V}$ and in the same way is calculated $\mathrm{C}_{\mathrm{T} 2}=0.024 \mathrm{~V}$. These results are close to the average of the experimental coefficients $C_{1}=0.0322 \mathrm{~V}$ and $C_{2}=0.0228 \mathrm{~V}$ correspondingly in part II from Figure 2.

The signal-to-noise ratio in decibels of the temporal pulse $\left(S N R_{t p, d B}\right)$ is calculated as

$$
S N R_{t p, d B}=10 \log _{10}\left(S N R_{t p}\right)
$$

where $S N R_{T P}$ is [11]

$$
S N R_{t p}=\int \frac{|f(t)|^{2}}{\left|f(t)-f^{*}(t)\right|^{2}} d t
$$

and $f^{*}(t)$ is the noiseless signal of the adjustment. The experimental curve of the temporal pulse for $C_{1}$ of Figure 3 has a $S N R_{t p, d B}=13.8 \mathrm{~dB}$.

The signal-to-noise ratio for a Fourier coefficient $C$ from a lock-in is calculated with the ratio of power of the signal $\left(P_{s}\right)$ and the power of the noise $\left(P_{n}\right)$ i.e., [12]

$$
S N R_{\text {lock-in }}=\frac{P_{S}}{P_{n}}
$$

where

$$
P_{s}=\frac{1}{2}|C|^{2} \text { and } P_{n}=\frac{K}{4 \tau}
$$

$K$ is the power density spectrum of the noise signal and $\tau$ is the time constant of the lock-in. $P_{n}$ shows the uncertainty of recovered coefficients of the lock-in, particularly with the time constant, hence, if $\tau$ is increased, the power of noise in the Fourier coefficient is decreased and its uncertainty is also decreased. It should be mentioned that this implies a compromise between the noise reduction of the Fourier coefficient and time of the measurement [12]. For measuring the dynamic of mist into the chamber $\tau=30 \mathrm{~s}$ and $\mathrm{K}$ is obtained through Parseval theorem [13]. The $S N R_{\text {lock-in,dB }}$ is obtained in the same way that $S N R_{t p, d B}$, therefor, for $C_{1}$ of the lock-in the $S N R_{\text {lock-in, } d B}=22 \mathrm{~dB}$. 
In order to analyze the behavior of Fourier coefficients respect to mist density, under the same parameters from the lock-in, backscattered light signals were obtained experimentally by the mist using a chamber length of $350 \mathrm{~cm}$. In Figure 4 it is shown the first coefficient $\left(C_{1}\right)$ and the second Fourier coefficient $\left(\mathrm{C}_{2}\right)$ of the backscattered pulse by the mist inside of the chamber corresponding to 150 and $350 \mathrm{~cm}$ of length. It can be notice that through the measurement of Fourier coefficients, it can be distinguished the effect of the mist density on the coefficients. As well, it is calculated the signal-to-noise ratio in decibels for a chamber length of $350 \mathrm{~cm}$, the $S N R_{t o, d B}$ of the temporal pulse is $12.3 \mathrm{~dB}$ and the $S N R_{\text {lock-in, } d B}$ for $\mathrm{C}_{1}$ is $20.4 \mathrm{~dB}$.

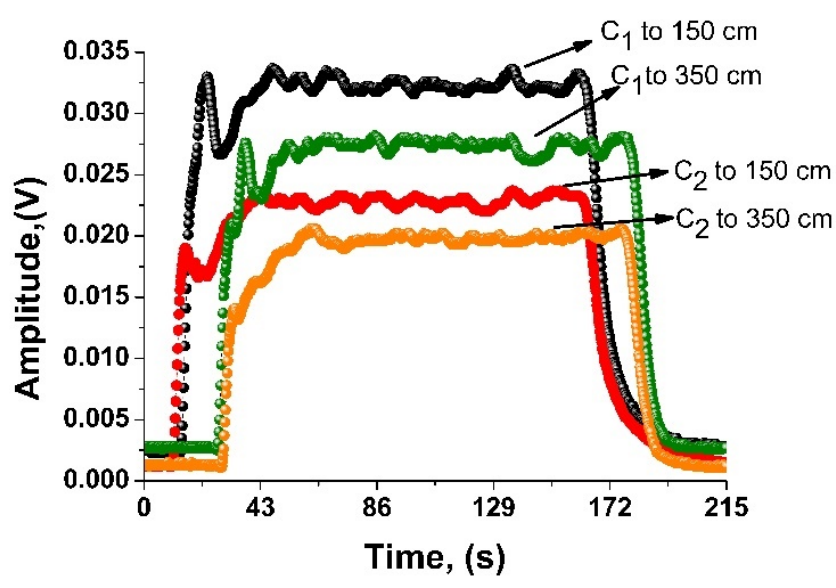

Figure 4. Fourier coefficients $C_{1}$ and $C_{2}$ corresponding to chamber lengths of 150 and $350 \mathrm{~cm}$.

Experimental and theoretical Fourier coefficients for the chamber lengths of 150 and $350 \mathrm{~cm}$ are shown in Figure 5. The orange bar is the experimental average value and the green is the value obtained from the corresponding adjustment for two lengths of the chamber with mist. The Figure 5 shows that the theoretical estimate is adequate for the experimental results considering a Gaussian function. For the case of the mist contained in the chamber with a length of $350 \mathrm{~cm}$, the theoretical FC moves away from the experimental value due to the fact that the temporal pulse is smaller in amplitude and noisier by the extinction of the pulse laser, but, the advantage of use the phase sensitive detection is the possibility of measure nosier signals $[12,15]$.

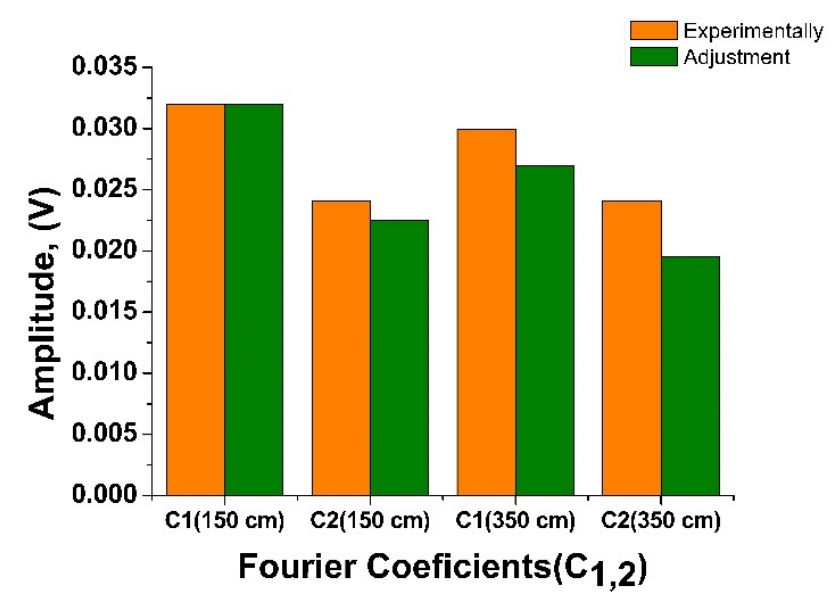

Figure 5. Experimental and theoretical Fourier coefficients.

\section{Conclusions}

In this work, it was shown that the technique of phase sensitive detection and SETS can be applied to process the LIDAR signal, this allowed to measure the Fourier coefficients with an improvement in 
the signal-to-noise ratio of the backscattered signal of a LIDAR system. The main advantage of use Fourier coefficients as a signal processing method is that for an input signal with a signal-to-noise ratio of $13.8 \mathrm{~dB}$, the signal-to-noise ratio of the first Fourier coefficient $C_{1}$ for a chamber length of $150 \mathrm{~cm}$ is $22 \mathrm{~dB}$; this corresponds to an improvement of approximately of $8 \mathrm{~dB}$, even more, the signal-to-noise ratio improvement was maintained with a different chamber length, this is due to the technique of phase sensitive detection can measure periodic signals into noise, similar improvement using EMD is shows by Chang, J. et al. [11]. The improvement for the two lengths of chamber shows the independence of the mist density and validate the improve of the LIDAR signal using Fourier coefficients. It should be mentioned that the OSLRF-01 system scales the speed of light approximately 881,000 times through sample time circuits (SETS) and this scaling allowed the use of a conventional lock-in as the SR530, however, in applications of short-range, atmospheric or other kind of LIDAR, the sensitive phase detection must be implemented with the correspond speed devices under the principle of SETS.

Author Contributions: J.A.D.-P. conceived the idea. All authors have jointly and equally made the work performed and approved the final version. All authors have read and agreed to the published version of the manuscript.

Funding: This research received no external funding.

Acknowledgments: Postdoctoral program PRODEP 511-6/17-14390.

Conflicts of Interest: The authors declare no conflict of interest.

\section{References}

1. Kumar, G.A.; Patil, A.; Patil, R.; Park, S.; Chai, Y. A LiDAR and IMU integrated indoor navigation system for UAVs and its application in real-time pipeline classification. Sensors 2017, 17, 1268. [CrossRef] [PubMed]

2. Jaboyedoff, M.; Oppikofer, T.; Abellán, A.; Derron, M.H.; Loye, A.; Metzger, R.; Pedrazzini, A. Use of LIDAR in landslide investigations: A review. Nat. Hazards 2012, 61, 5-28. [CrossRef]

3. Weitkamp, C. (Ed.) Lidar: Range-Resolved Optical Remote Sensing of the Atmosphere; Springer Science \& Business: Berlin/Heidelberg, Germany, 2006; Volume 102.

4. Dwivedi, Y.; Rai, S.B.; Singh, J.P. Spectroscopic Techniques for Security, Forensic and Environmental Applications, Chemistry Research and Applications; Nova Science Publishers Incorporated: New York, NY, USA, 2014.

5. Castrejón-García, R.; Varela, J.R.; Castrejón-Pita, J.R.; Morales, A. The laser-backscattering equations and their application to the study of the atmospheric structure. Rev. Mex. Física 2002, 48, 513-518.

6. Castrejón-García, R.; Varela, J.R.; Hernández Utrera, O.; Altamirano-Robles, L. Design and development of an elastic-scattering lidar for the study of the atmospheric structure. Rev. Mex. Física 2017, 63, 49-54.

7. Rairoux, P.; Schillinger, H.; Niedermeier, S.; Rodriguez, M.; Ronneberger, F.; Sauerbrey, R.; Wöste, L. Remote sensing of the atmosphere using ultrashort laser pulses. Appl. Phys. B 2000, 71, 573-580. [CrossRef]

8. Kashani, A.G.; Olsen, M.J.; Parrish, C.E.; Wilson, N. A review of LIDAR radiometric processing: From ad hoc intensity correction to rigorous radiometric calibration. Sensors 2015, 15, 28099-28128. [CrossRef] [PubMed]

9. Wu, S.; Liu, Z.; Liu, B. Enhancement of lidar backscatters signal-to-noise ratio using empirical mode decomposition method. Opt. Commun. 2006, 267, 137-144. [CrossRef]

10. Tian, P.; Cao, X.; Liang, J.; Zhang, L.; Yi, N.; Wang, L.; Cheng, X. Improved empirical mode decomposition based denoising method for lidar signals. Opt. Commun. 2014, 325, 54-59. [CrossRef]

11. Chang, J.; Zhu, L.; Li, H.; Xu, F.; Liu, B.; Yang, Z. Noise reduction in Lidar signal using correlation-based EMD combined with soft thresholding and roughness penalty. Opt. Commun. 2018, 407, 290-295. [CrossRef]

12. Dávila Pintle, J.A. Fourier description of lock-in. Rev. Mex. Física E 2013, 59, 1-7.

13. Lathi, B.P. Signals, Systems and Communication; John Wiley and Sons: Hoboken, NJ, USA, 1965.

14. Hwei, P.H. Fourier Analysis; Simon and Shuster: New York, NY, USA, 1970.

15. Meade, M.L. Lock-In Amplifiers: Principles and Applications (No. 1); Peter Peregrinus Ltd.: London, UK, 1983. 